\title{
Polysubstituted Ligand Framework for Color Tuning Phosphorescent Iridium(III) Complexes
}

Sophie A. Fitzgerald, ${ }^{a}$ Haleema Y. Otaif, ${ }^{a}$ Christopher E. Elgar, ${ }^{a}$ Natalia Sawicka, ${ }^{a}$ Peter N. Horton, ${ }^{b}$ Simon J. Coles, ${ }^{b}$ Joseph M. Beames, ${ }^{a}$ and Simon J.A. Pope*a

aSchool of Chemistry, Main Building, Cardiff University, Cardiff CF10 3AT, Cymru/Wales, UK; bUK National Crystallographic Service, Chemistry, Faculty of Natural and Environmental Sciences, University of Southampton, Highfield, Southampton, SO17 1BJ, England, UK. Email: popesj@cardiff.ac.uk

\section{Supporting Information}

\section{Contents}

Table S1 pS-2

Table S2 pS-3

Figure S1 pS-4

Figure S2 pS-5

Figure S3 pS-6

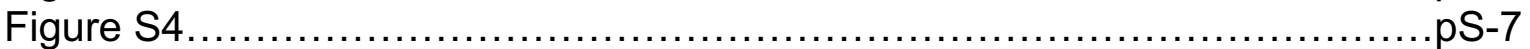

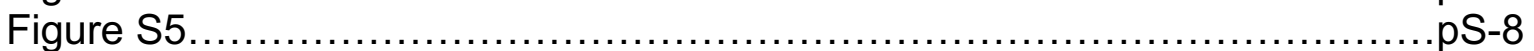

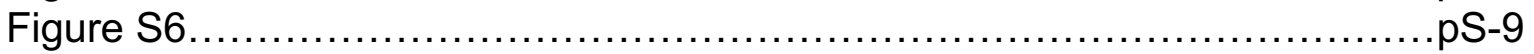

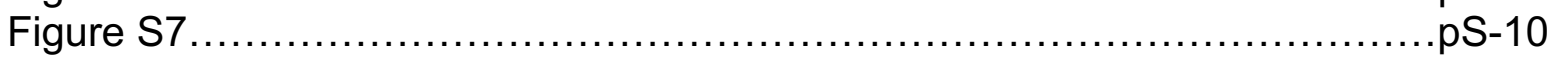

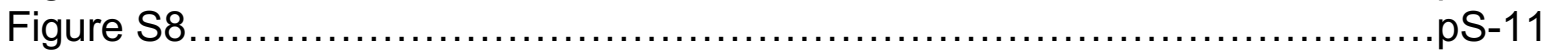

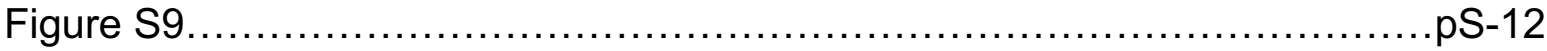

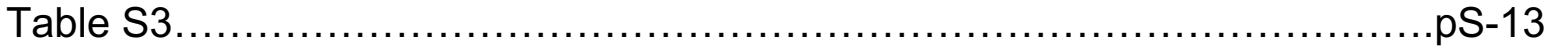

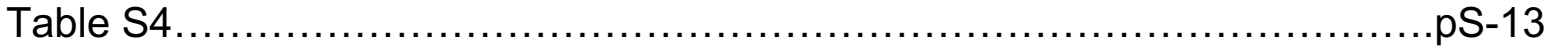

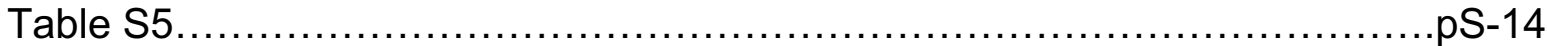

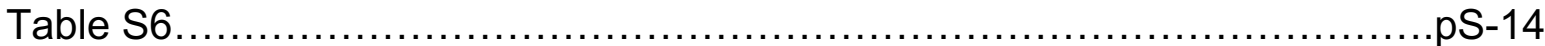

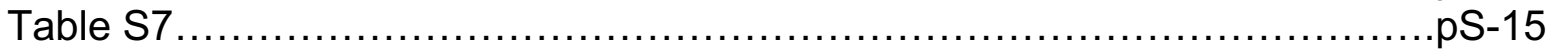

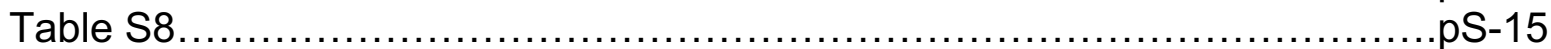

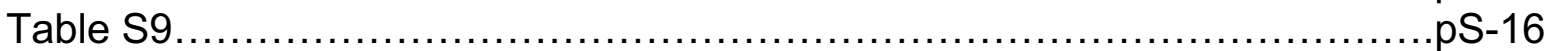

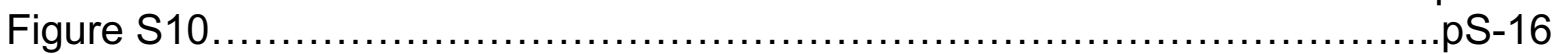

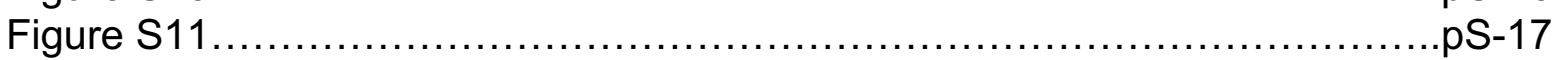

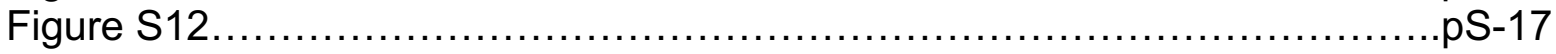

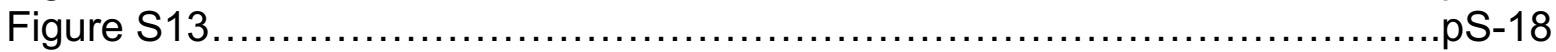

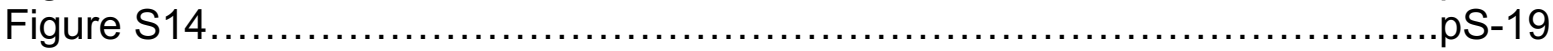

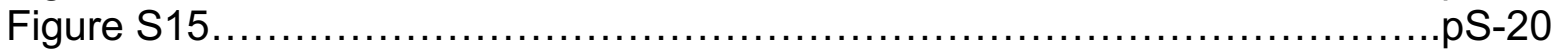


Table S1. Data collection parameters for the X-ray crystal structures.

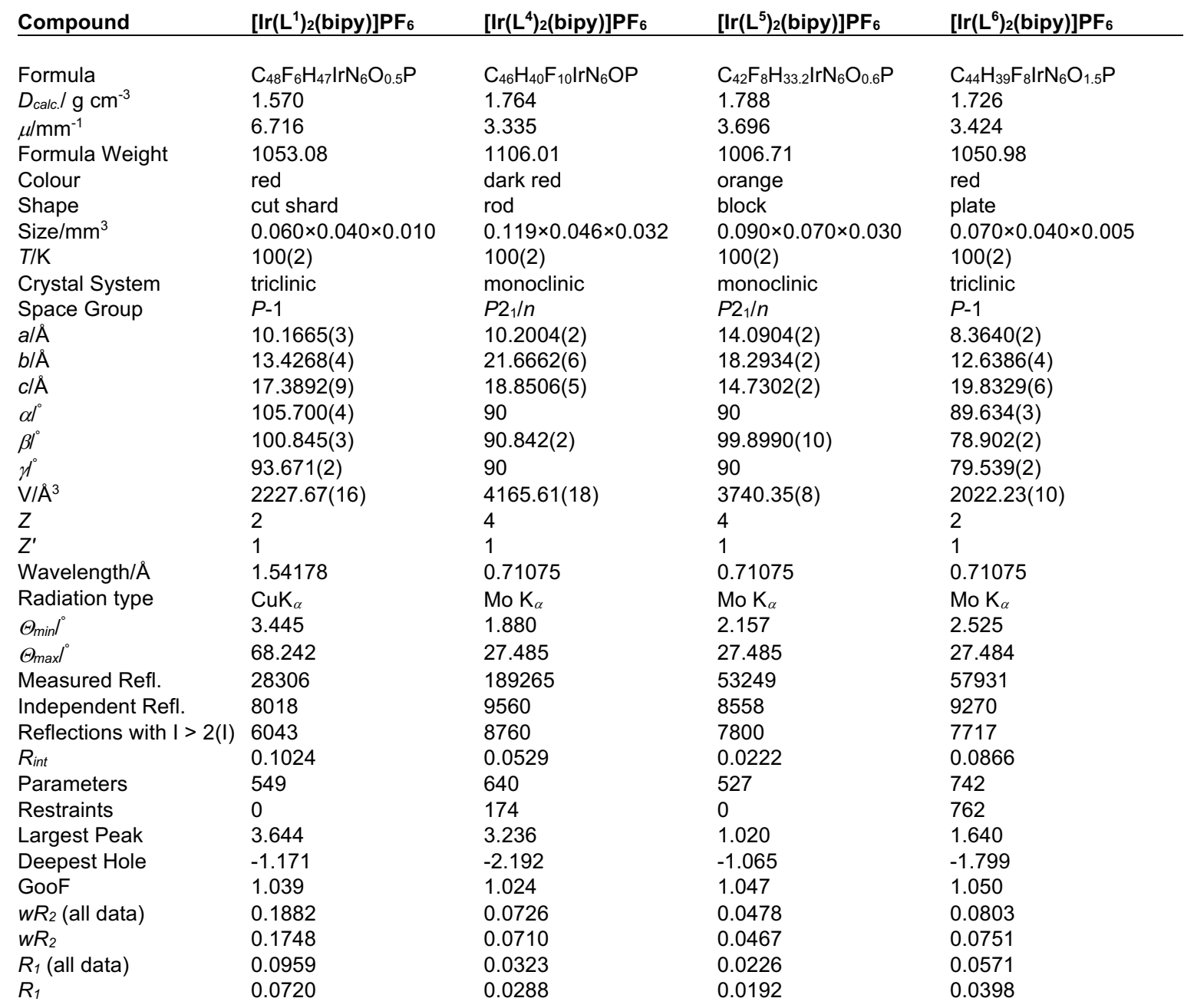


Table S2. Selected bond lengths $(\AA)$ and bond angles $\left({ }^{\circ}\right)$ from the crystallographic data.

$\left[\operatorname{lr}\left(\mathrm{L}^{1}\right)_{2}(\mathrm{bipy})\right] \mathrm{PF} \mathrm{F}_{6}$

$\left[\operatorname{lr}\left(\mathrm{L}^{4}\right)_{2}(\mathrm{bipy})\right] \mathrm{PF}_{6}$

$\left[\operatorname{lr}\left(\mathbf{L}^{5}\right)_{2}(\right.$ bipy $\left.)\right] \mathrm{PF}_{6}$

$\left[\operatorname{Ir}\left(\mathbf{L}^{7}\right)_{2}(\right.$ bipy $\left.)\right] \mathrm{PF}_{6}$

\begin{tabular}{|c|c|c|c|c|c|c|c|}
\hline \multicolumn{2}{|c|}{ Bond lengths $(\AA)$} & \multicolumn{2}{|c|}{ Bond lengths $(\AA)$} & \multicolumn{2}{|c|}{ Bond lengths $(\AA)$} & \multicolumn{2}{|c|}{ Bond lengths $(\AA)$} \\
\hline $\operatorname{Ir}(1)-\mathrm{N}(1)$ & $2.100(8)$ & $\operatorname{Ir}(1)-\mathrm{N}(1)$ & $2.079(3)$ & $\operatorname{Ir}(1)-\mathrm{N}(1)$ & $2.0890(18)$ & $\operatorname{Ir}(1)-\mathrm{N}(1)$ & $2.068(4)$ \\
\hline $\operatorname{Ir}(1)-N(21)$ & $2.090(8)$ & $\operatorname{Ir}(1)-\mathrm{N}(21)$ & $2.080(3)$ & $\operatorname{Ir}(1)-\mathrm{N}(21)$ & $2.093(2)$ & $\operatorname{Ir}(1)-\mathrm{N}(42)$ & $2.159(4)$ \\
\hline $\operatorname{Ir}(1)-N(41)$ & $2.176(8)$ & $\operatorname{Ir}(1)-N(41)$ & $2.161(3)$ & $\operatorname{Ir}(1)-N(41)$ & $2.1653(19)$ & $\operatorname{Ir}(1)-N(21)$ & $2.073(4)$ \\
\hline $\operatorname{Ir}(1)-N(42)$ & $2.148(8)$ & $\operatorname{Ir}(1)-\mathrm{N}(42)$ & $2.167(3)$ & $\operatorname{Ir}(1)-\mathrm{N}(42)$ & $2.1412(19)$ & $\operatorname{Ir}(1)-\mathrm{N}(41)$ & $2.191(4)$ \\
\hline $\operatorname{Ir}(1)-C(1)$ & $2.023(9)$ & $\operatorname{Ir}(1)-C(1)$ & $1.992(3)$ & $\operatorname{Ir}(1)-C(1)$ & $2.006(2)$ & $\operatorname{Ir}(1)-C(1)$ & $1.992(5)$ \\
\hline $\operatorname{Ir}(1)-C(21)$ & $1.974(9)$ & $\operatorname{Ir}(1)-C(21)$ & $2.017(3)$ & $\operatorname{Ir}(1)-C(21)$ & $2.009(2)$ & $\operatorname{Ir}(1)-C(21)$ & $2.009(4)$ \\
\hline \multicolumn{2}{|c|}{ Bond angles $\left({ }^{\circ}\right)$} & \multicolumn{2}{|c|}{ Bond angles $\left({ }^{\circ}\right)$} & \multicolumn{2}{|c|}{ Bond angles $\left({ }^{\circ}\right)$} & \multicolumn{2}{|c|}{ Bond angles $\left({ }^{\circ}\right)$} \\
\hline$N(1)-\operatorname{Ir}(1)-N(41)$ & $105.5(3)$ & $N(1)-\operatorname{Ir}(1)-N(21)$ & $173.30(10)$ & $N(1)-\operatorname{Ir}(1)-N(21)$ & 171.01(7) & $N(1)-\operatorname{Ir}(1)-N(42)$ & $85.55(14)$ \\
\hline$N(1)-\operatorname{Ir}(1)-N(42)$ & $84.8(3)$ & $N(1)-\operatorname{Ir}(1)-N(41)$ & $102.99(10)$ & $N(1)-\operatorname{Ir}(1)-N(41)$ & $105.77(7)$ & $N(1)-\operatorname{Ir}(1)-N(1)$ & $171.30(15)$ \\
\hline$N(21)-\operatorname{Ir}(1)-N(1)$ & $171.9(3)$ & $N(1)-\operatorname{Ir}(1)-N(42)$ & $83.70(10)$ & $N(1)-\operatorname{Ir}(1)-N(42)$ & $85.44(7)$ & $N(1)-\operatorname{Ir}(1)-N(21)$ & $106.77(15)$ \\
\hline$N(21)-\operatorname{Ir}(1)-N(41)$ & $81.2(3)$ & $N(21)-\operatorname{Ir}(1)-N(41)$ & $80.83(10)$ & $N(21)-\operatorname{Ir}(1)-N(41)$ & $81.15(7)$ & $N(42)-\operatorname{Ir}(1)-N(41)$ & $75.04(15)$ \\
\hline$N(21)-\operatorname{Ir}(1)-N(42)$ & $101.5(3)$ & $N(21)-\operatorname{Ir}(1)-N(42)$ & $102.66(11)$ & $N(21)-\operatorname{Ir}(1)-N(42)$ & $102.00(8)$ & $N(21)-\operatorname{Ir}(1)-N(42)$ & $102.02(15)$ \\
\hline$N(42)-\operatorname{Ir}(1)-N(41)$ & $75.3(3)$ & $N(42)-\operatorname{Ir}(1)-N(41)$ & $75.93(10)$ & $N(42)-\operatorname{Ir}(1)-N(41)$ & $76.03(7)$ & $N(21)-\operatorname{Ir}(1)-N(41)$ & 79.61(15) \\
\hline$C(1)-\operatorname{Ir}(1)-N(1)$ & $79.4(4)$ & $C(1)-\operatorname{Ir}(1)-N(1)$ & $79.50(12)$ & $C(1)-\operatorname{Ir}(1)-N(1)$ & $79.54(8)$ & $C(1)-\operatorname{Ir}(1)-N(1)$ & $79.77(16)$ \\
\hline$C(1)-\operatorname{Ir}(1)-N(21)$ & $94.6(4)$ & $C(1)-\operatorname{Ir}(1)-N(21)$ & $97.76(12)$ & $C(1)-\operatorname{Ir}(1)-N(21)$ & $94.32(8)$ & $C(1)-\operatorname{Ir}(1)-N(42)$ & $95.87(16)$ \\
\hline$C(1)-\operatorname{Ir}(1)-N(41)$ & $171.3(3)$ & $C(1)-\operatorname{Ir}(1)-N(41)$ & $169.35(11)$ & $C(1)-\operatorname{Ir}(1)-N(41)$ & $171.33(8)$ & $C(1)-\operatorname{Ir}(1)-N(21)$ & $95.04(17)$ \\
\hline$C(1)-\operatorname{Ir}(1)-N(42)$ & $98.3(3)$ & $C(1)-\operatorname{Ir}(1)-N(42)$ & $94.19(11)$ & $C(1)-\operatorname{Ir}(1)-N(42)$ & $97.86(8)$ & $C(1)-\operatorname{Ir}(1)-N(41)$ & $168.05(16)$ \\
\hline$C(21)-\operatorname{Ir}(1)-N(1)$ & $94.1(3)$ & $C(1)-\operatorname{Ir}(1)-C(21)$ & $93.78(13)$ & $C(1)-\operatorname{Ir}(1)-N(21)$ & $88.91(9)$ & $C(1)-\operatorname{Ir}(1)-C(21)$ & $92.93(17)$ \\
\hline$C(21)-\operatorname{Ir}(1)-N(21)$ & $80.5(3)$ & $C(21)-\operatorname{Ir}(1)-N(1)$ & $94.65(12)$ & $C(21)-\operatorname{Ir}(1)-N(1)$ & $93.10(9)$ & $C(21)-\operatorname{Ir}(1)-N(1)$ & $93.25(16)$ \\
\hline$C(21)-\operatorname{Ir}(1)-N(41)$ & $96.0(3)$ & $C(21)-\operatorname{Ir}(1)-N(21)$ & $79.37(13)$ & $C(21)-\operatorname{Ir}(1)-N(21)$ & $80.13(9)$ & $C(21)-\operatorname{Ir}(1)-N(42)$ & $170.75(15)$ \\
\hline$C(21)-\operatorname{Ir}(1)-N(42)$ & $170.5(3)$ & $C(21)-\operatorname{Ir}(1)-N(41)$ & $96.31(12)$ & $C(21)-\operatorname{Ir}(1)-N(41)$ & $97.53(8)$ & $C(21)-\operatorname{Ir}(1)-N(21)$ & $79.96(17)$ \\
\hline$C(21)-\operatorname{Ir}(1)-C(1)$ & $90.8(3)$ & $C(21)-\operatorname{Ir}(1)-N(42)$ & $171.41(12)$ & $C(21)-\operatorname{Ir}(1)-N(42)$ & $172.69(8)$ & $C(21)-\operatorname{Ir}(1)-N(41)$ & $96.60(17)$ \\
\hline
\end{tabular}




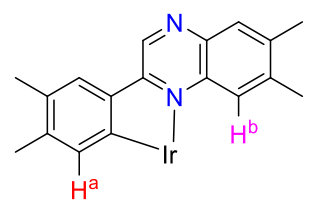

6.70 ppm

(s, 2H)

6.14 ppm

(s, 2H)

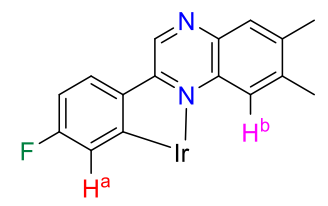

6.78 ppm

(s, 2H)

6.19 ppm

(dd, ${ }^{3} \mathrm{~J}_{\mathrm{HF}}=9.5 \mathrm{~Hz},{ }^{4} \mathrm{~J}_{\mathrm{HH}}=2.3 \mathrm{~Hz}, 2 \mathrm{H}$ )

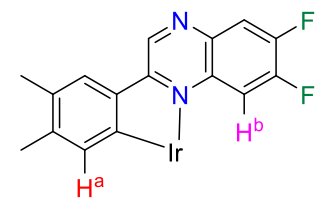

6.73 ppm

$\left(\mathrm{dd},{ }^{3} \mathrm{~J}_{\mathrm{HF}}=11.8 \mathrm{~Hz},{ }^{4} \mathrm{~J}_{\mathrm{HF}}=7.5 \mathrm{~Hz}, 2 \mathrm{H}\right)$

$6.12 \mathrm{ppm}$

(s, 2H)

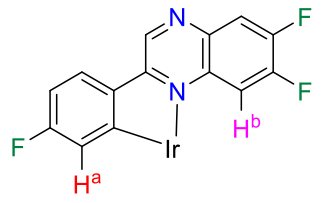

6.80 ppm

(dd, ${ }^{3} \mathrm{~J}_{\mathrm{HF}}=12.4 \mathrm{~Hz},{ }^{4} \mathrm{~J}_{\mathrm{HF}}=7.8 \mathrm{~Hz}, 2 \mathrm{H}$ )

$6.29 \mathrm{ppm}$

(dd, ${ }^{3} \mathrm{~J}_{\mathrm{HF}}=9.5 \mathrm{~Hz},{ }^{4} \mathrm{~J}_{\mathrm{HH}}=2.5 \mathrm{~Hz}, 2 \mathrm{H}$ )

Figure S1. A comparison of signature upfield ${ }^{1} \mathrm{H}$ resonances for selected $\operatorname{Ir}(\mathrm{III})$ coordinated 2-pqx ligands (clockwise from top left $\mathbf{L}^{1}, \mathbf{L}^{2}, \mathbf{L}^{7}, \mathbf{L}^{6}$ ). 
30-Mar-2021

SP MS35384 ESP $25(0.519)$

100

100

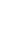

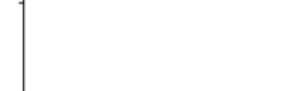

ㅇำ

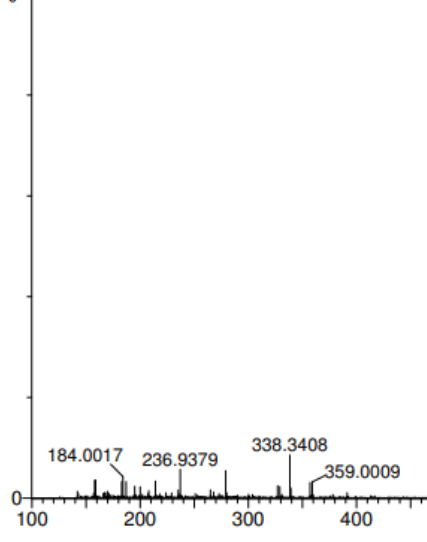

Figure S2. Example HRMS spectrum of $\left[\operatorname{Ir}\left(\mathrm{L}^{9}\right)_{2}(\right.$ bipy $\left.)\right] \mathrm{PF}_{6}$ showing the parent cation. 

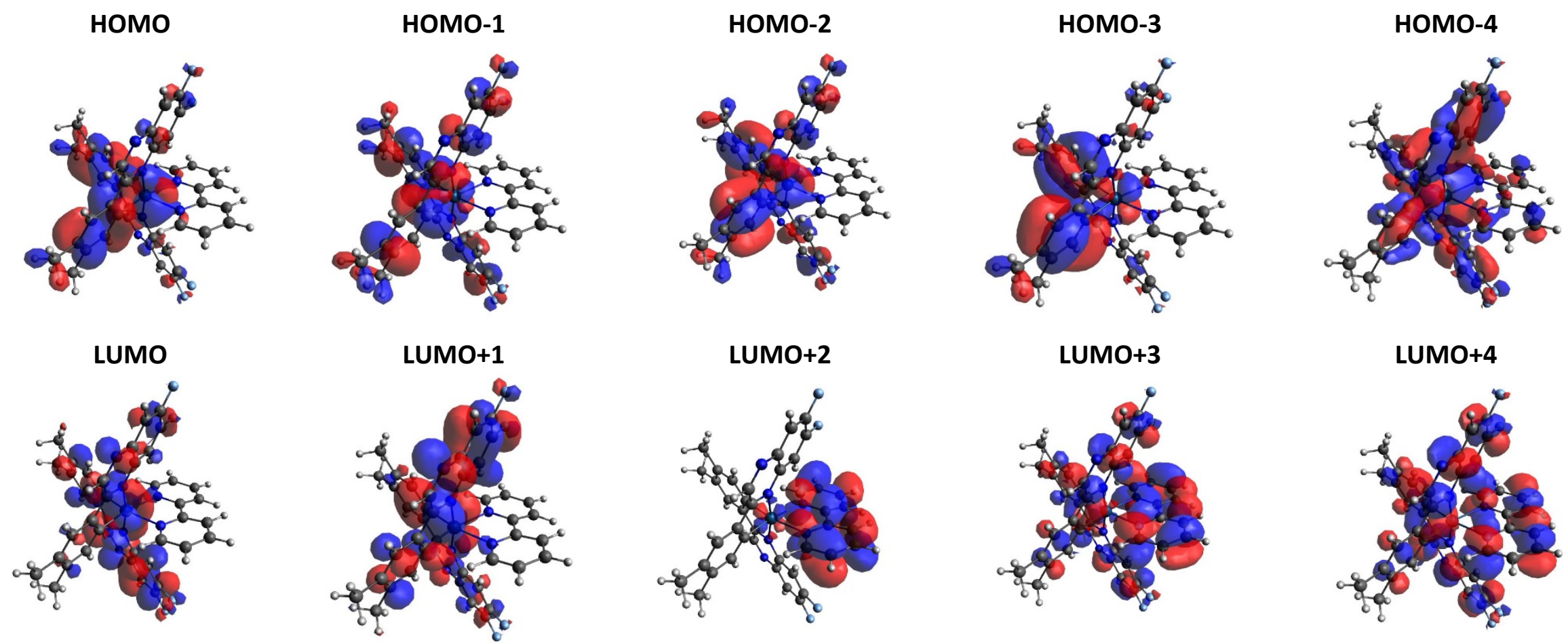

Figure S3. Calculated Kohn-Sham frontier molecular orbitals for $\left[\operatorname{Ir}\left(\mathrm{L}^{2}\right)_{2}(\text { bipy })\right]^{+}$. 

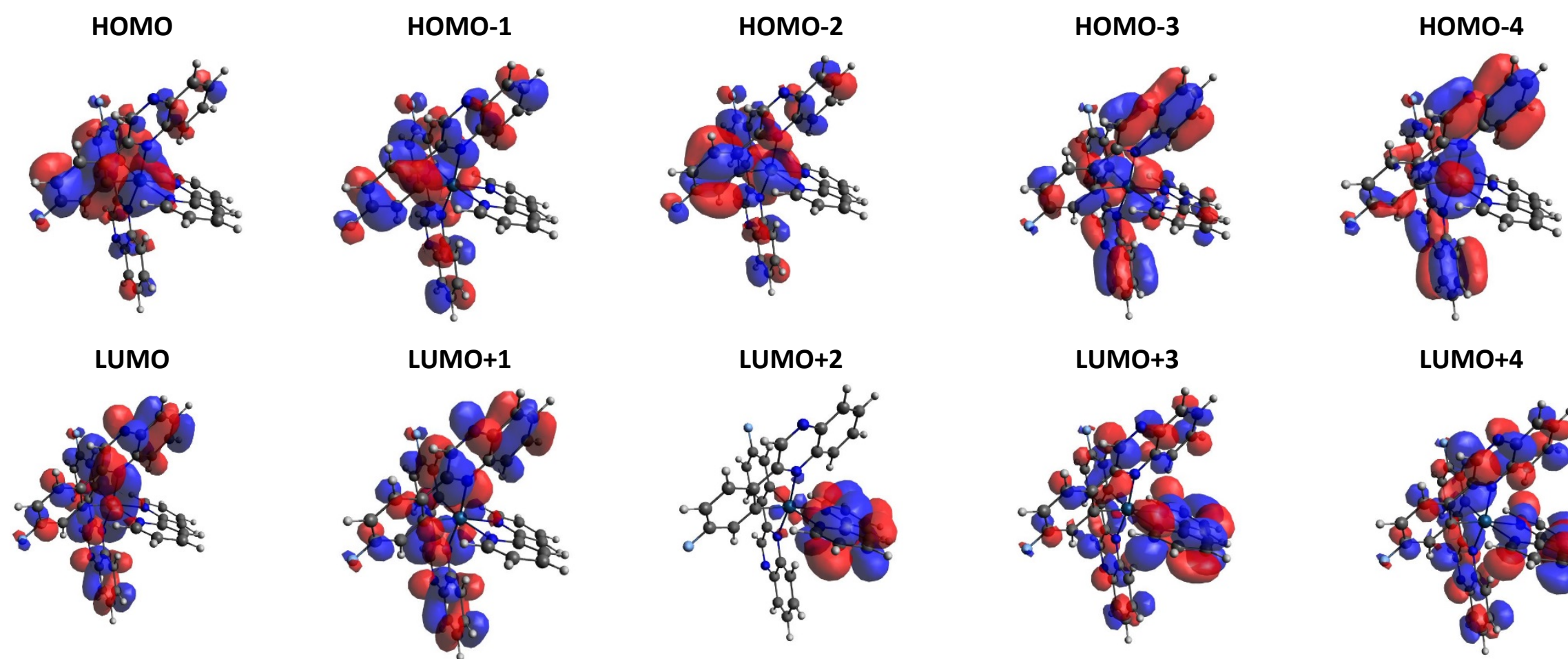

Figure S4. Calculated Kohn-Sham frontier molecular orbitals for $\left[\operatorname{Ir}\left(\mathbf{L}^{5}\right)_{2}(\text { bipy })\right]^{+}$. 

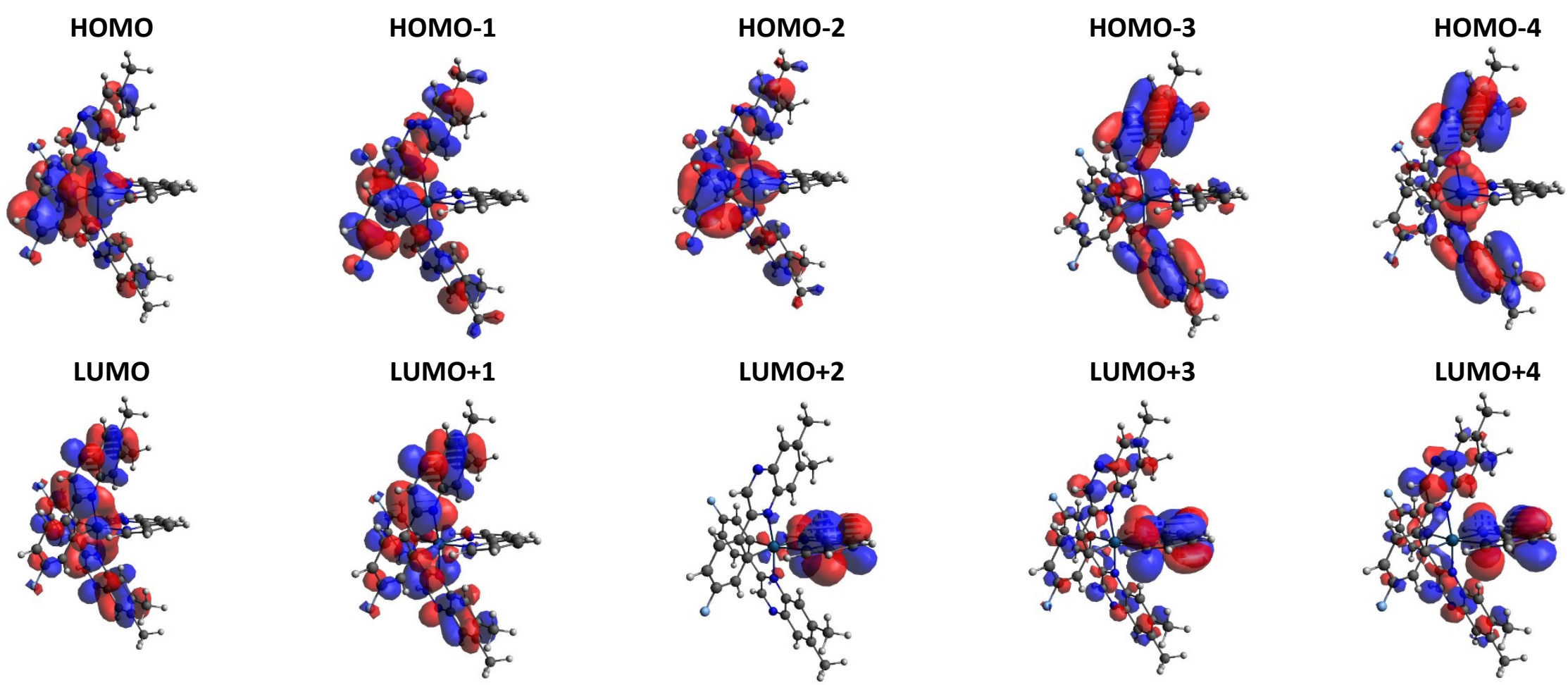

Figure S5. Calculated Kohn-Sham frontier molecular orbitals for $\left[\operatorname{lr}\left(\operatorname{L}^{6}\right)_{2}(\text { bipy })\right]^{+}$. 

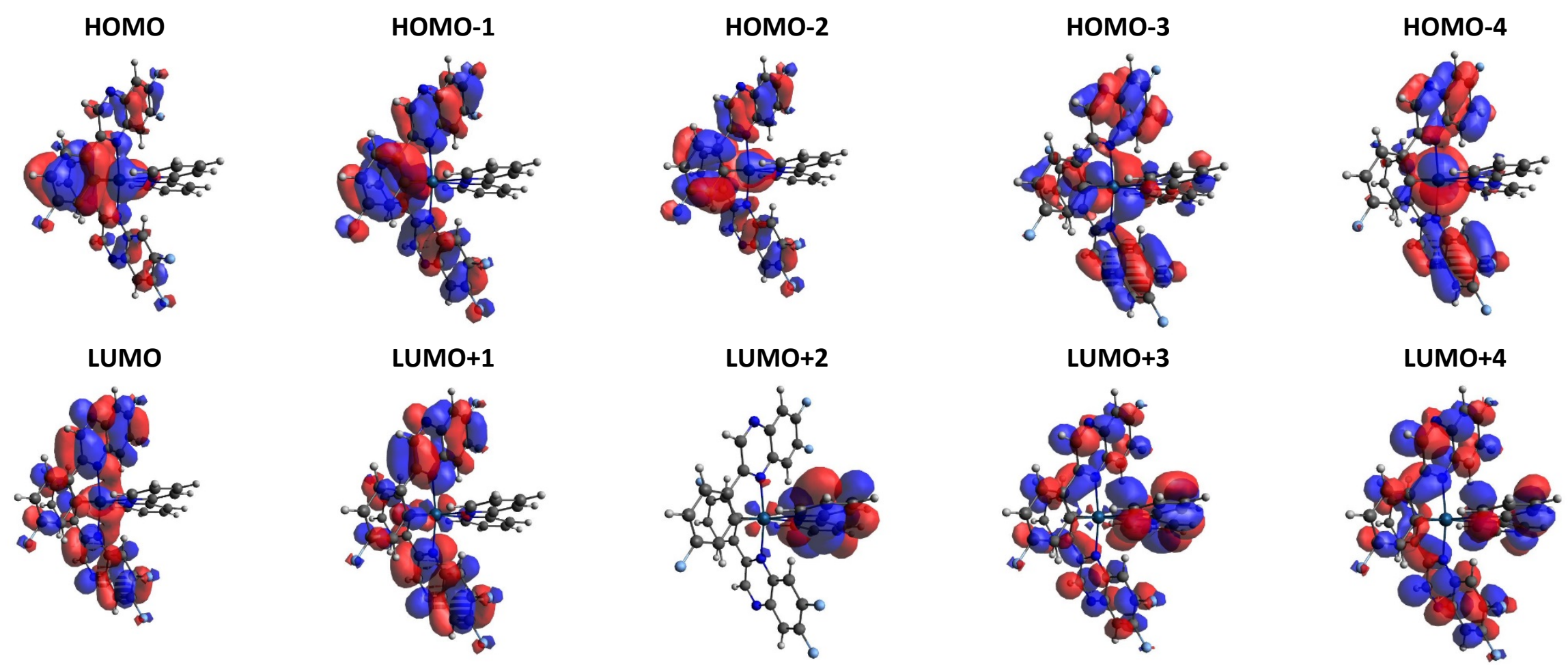

Figure S6. Calculated Kohn-Sham frontier molecular orbitals for $\left[\operatorname{lr}\left(\mathbf{L}^{7}\right)_{2}(\text { bipy })\right]^{+}$. 

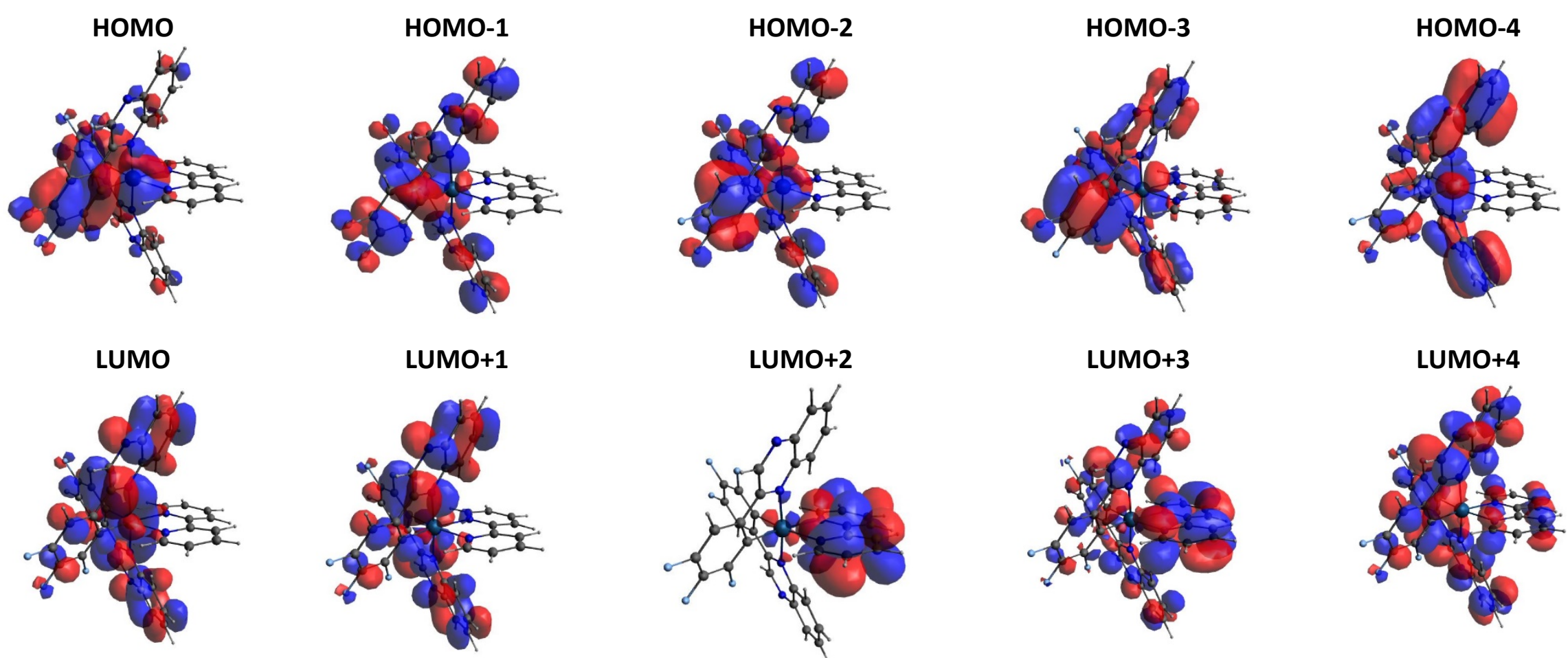

Figure S7. Calculated Kohn-Sham frontier molecular orbitals for $\left[\operatorname{Ir}\left(\mathbf{L}^{8}\right)_{2}(\text { bipy })\right]^{+}$. 

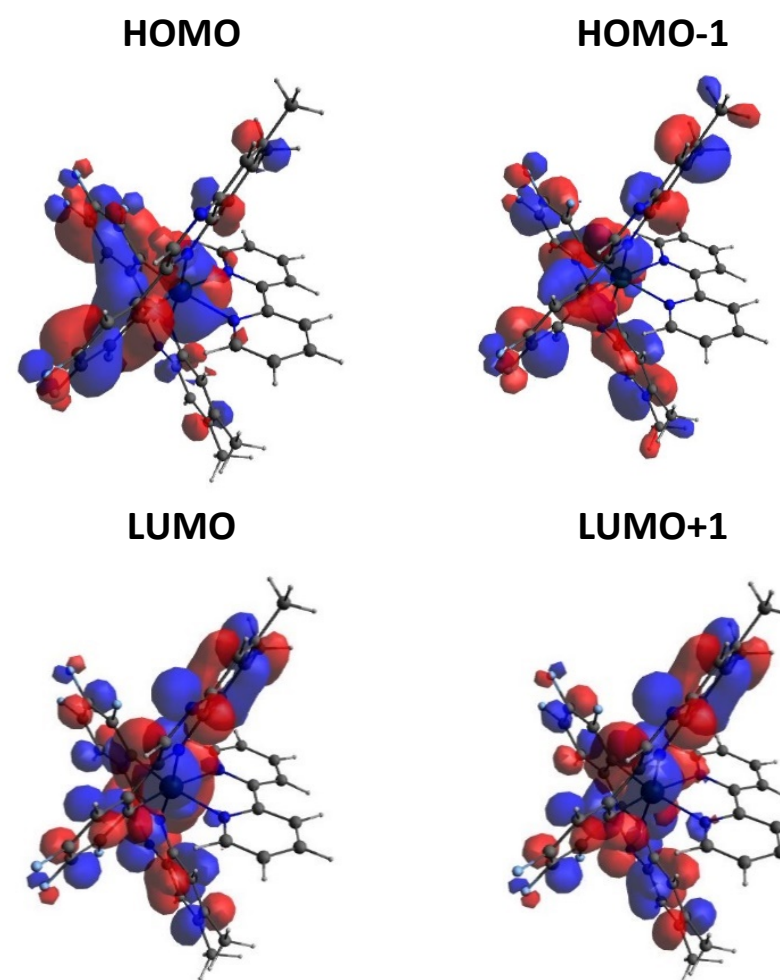

HOMO-1

LUMO+1

HOMO-2

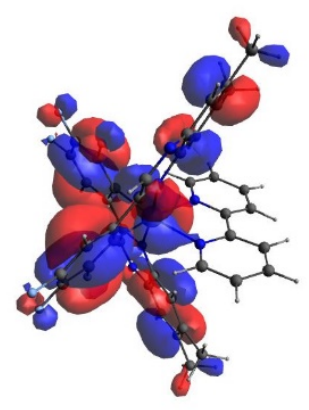

LUMO+2

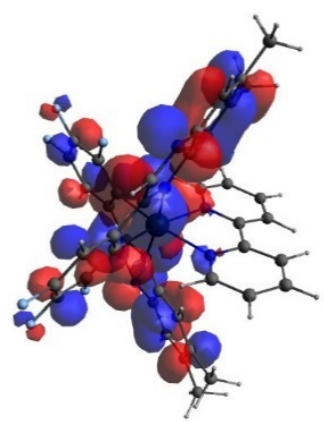

HOMO-3

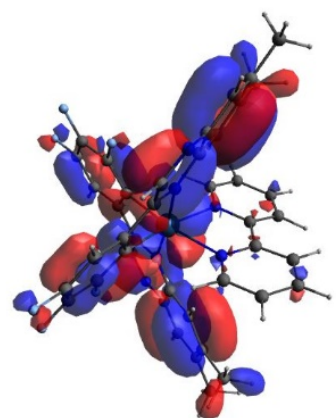

LUMO+3
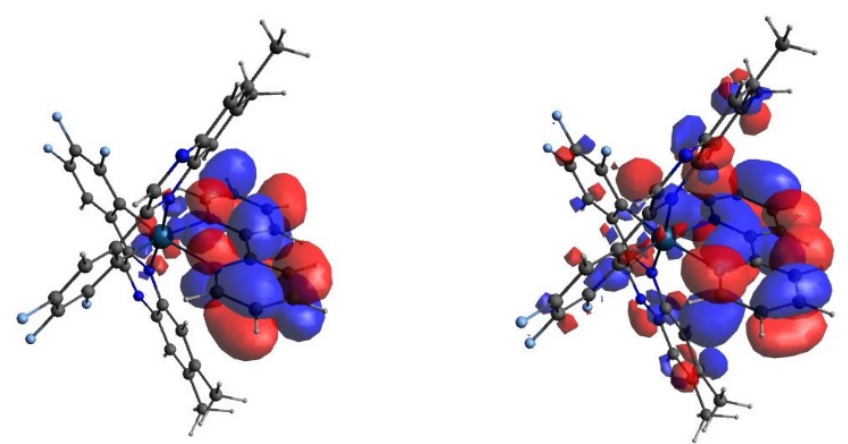

HOMO-4

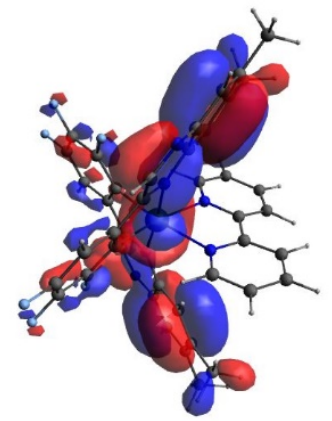

LUMO+4

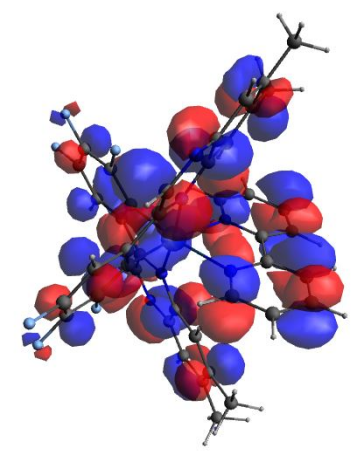

Figure S8. Calculated Kohn-Sham frontier molecular orbitals for $\left[\operatorname{Ir}\left(\mathrm{L}^{9}\right)_{2}(\text { bipy })\right]^{+}$. 

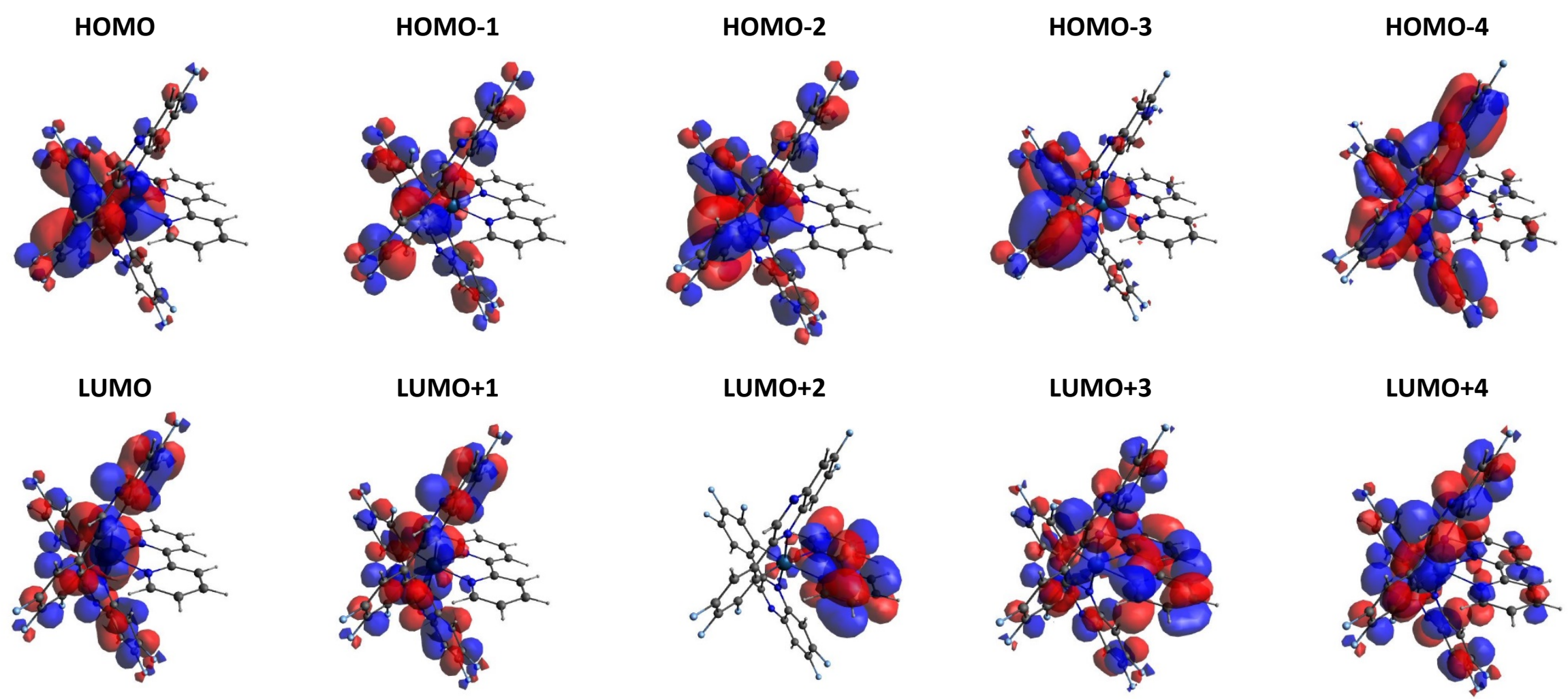

Figure S9. Calculated Kohn-Sham frontier molecular orbitals for $\left[\operatorname{Ir}\left(\mathrm{L}^{10}\right)_{2}(\text { bipy })\right]^{+}$. 
Table S3. A description of the calculated MO contributions, excited state descriptions and their associated transitions for $\left[\operatorname{lr}\left(\mathrm{L}^{\mathbf{2}}\right)_{2}(\text { bipy })\right]^{+}$complex $(\mathrm{Q} 1$ and $\mathrm{Q} 2$ are the different quinoxaline ligands; bipy = bipyridine).

\begin{tabular}{|c|c|c|c|c|c|c|}
\hline \multirow[b]{2}{*}{ Orbital } & \multicolumn{4}{|c|}{$\begin{array}{c}\text { Moiety Contribution to } \\
\text { Orbital (\%) }\end{array}$} & \multicolumn{2}{|c|}{ Orbital Contribution to Excited State } \\
\hline & $\operatorname{Ir}(5 d)$ & bipy & Q1 & Q2 & Excited State & Contributing Transitions (> 10\%) \\
\hline LUMO +4 & 1 & 38 & 30 & 30 & $1(419 \mathrm{~nm} \mathrm{f}=0.1768)$ & HOMO $\rightarrow$ LUMO+1 (84\%) \\
\hline LUMO +3 & 2 & 60 & 19 & 19 & & \\
\hline LUMO +2 & 2 & 96 & 1 & 1 & $2(412 \mathrm{~nm} f=0.0456)$ & HOMO-1 $\rightarrow$ LUMO+1 (11\%) \\
\hline LUMO +1 & 4 & 1 & 32 & 64 & & HOMO $\rightarrow$ LUMO (84\%) \\
\hline LUMO & 4 & 1 & 63 & 31 & & \\
\hline HOMO & 32 & 2 & 33 & 33 & $3(341 \mathrm{~nm} f=0.0578)$ & $\mathrm{HOMO} \rightarrow \mathrm{LUMO}+2(78 \%)$ \\
\hline HOMO -1 & 4 & 1 & 47 & 47 & & \\
\hline HOMO -2 & 8 & 1 & 45 & 46 & $4(336 \mathrm{~nm} f=0.1809)$ & HOMO $-2 \rightarrow$ LUMO (24\%) \\
\hline HOMO -3 & 12 & 2 & 43 & 43 & & HOMO -1 $\rightarrow$ LUMO +1(59\%) \\
\hline HOMO -4 & 44 & 8 & 24 & 24 & $5(334 \mathrm{~nm} \mathrm{f}=0.2519)$ & $\begin{array}{l}\text { HOMO }-2 \rightarrow \text { LUMO+1 (21\%) } \\
\text { HOMO-1 } \rightarrow \text { LUMO (49\%) } \\
\text { HOMO } \rightarrow \text { LUMO+2 (13\%) }\end{array}$ \\
\hline
\end{tabular}

Table S4. A description of the calculated MO contributions, excited state descriptions and their associated transitions for $\left[\operatorname{Ir}\left(\mathbf{L}^{\mathbf{5}}\right)_{2}(\text { bipy })\right]^{+}$complex $(\mathrm{Q} 1$ and $\mathrm{Q} 2$ are the different quinoxaline ligands; bipy = bipyridine).

\begin{tabular}{|c|c|c|c|c|c|c|}
\hline \multirow[b]{2}{*}{ Orbital } & \multicolumn{4}{|c|}{$\begin{array}{c}\text { Moiety Contribution to } \\
\text { Orbital (\%) }\end{array}$} & \multicolumn{2}{|c|}{ Orbital Contribution to Excited State } \\
\hline & $\operatorname{Ir}(5 d)$ & bipy & L1 & $\mathbf{L 2}$ & Excited State & Contributing Transitions (> 10\%) \\
\hline LUMO +4 & 1 & 44 & 28 & 27 & $1(385 \mathrm{~nm} f=0.1896)$ & HOMO-1 $\rightarrow$ LUMO (12\%) \\
\hline LUMO +3 & 2 & 64 & 17 & 17 & & HOMO $\rightarrow$ LUMO+1 (81\%) \\
\hline LUMO +2 & 2 & 96 & 1 & 1 & $2(380 \mathrm{~nm} f=0.0590)$ & HOMO-1 $\rightarrow$ LUMO+1 (14\%) \\
\hline LUMO +1 & 4 & 1 & 48 & 48 & & HOMO $\rightarrow$ LUMO (81\%) \\
\hline LUMO & 4 & 1 & 48 & 47 & & \\
\hline HOMO & 35 & 2 & 32 & 32 & $3(322 \mathrm{~nm}$ f=0.1107) & HOMO-2 $\rightarrow$ LUMO+1 (14\%) \\
\hline HOMO -1 & 8 & 1 & 46 & 46 & & $\begin{array}{l}\text { HOMO-1 } \rightarrow \text { LUMO (29\%) } \\
\text { HOMO } \rightarrow \text { LUMO+2 (43\%) }\end{array}$ \\
\hline HOMO -2 & 14 & 1 & 43 & 42 & $4(319 \mathrm{~nm} \quad \mathrm{f}=0.1216)$ & HOMO-2 $\rightarrow$ LUMO (25\%) \\
\hline HOMO -3 & 38 & 6 & 28 & 28 & & HOMO-1 $\rightarrow$ LUMO+1(53\%) \\
\hline HOMO -4 & 33 & 2 & 33 & 32 & $5(318 \mathrm{~nm} \quad \mathrm{f}=0.0666)$ & $\begin{array}{l}\text { HOMO }-2 \rightarrow \text { LUMO+1 (10\%) } \\
\text { HOMO-1 } \rightarrow \text { LUMO }(24 \%) \\
\text { HOMO } \rightarrow \text { LUMO+2 (45\%) }\end{array}$ \\
\hline
\end{tabular}


Table S5. A description of the calculated MO contributions, excited state descriptions and their associated transitions for $\left[\operatorname{Ir}\left(\mathrm{L}^{6}\right)_{2}(\text { bipy })\right]^{+}$complex $(\mathrm{Q} 1$ and $\mathrm{Q} 2$ are the different quinoxaline ligands; bipy = bipyridine).

\begin{tabular}{|c|c|c|c|c|c|c|}
\hline & \multicolumn{4}{|c|}{ Moiety Contribution to Orbital (\%) } & \multicolumn{2}{|c|}{ Orbital Contribution to Excited State } \\
\hline Orbital & $\operatorname{Ir}(5 d)$ & bipy & Q1 & Q2 & Excited State & Contributing Transitions (> 10\%) \\
\hline LUMO +4 & 1 & 70 & 14 & 14 & $1(383 \mathrm{~nm} \mathrm{f}=0.2536)$ & HOMO-1 $\rightarrow$ LUMO (14\%) \\
\hline LUMO +3 & 2 & 76 & 11 & 11 & & HOMO $\rightarrow$ LUMO+1 (78\%) \\
\hline LUMO +2 & 2 & 96 & 1 & 1 & $2(379 \mathrm{~nm} f=0.0860)$ & HOMO-1 $\rightarrow$ LUMO+1 (17\%) \\
\hline LUMO +1 & 4 & 2 & 45 & 50 & & HOMO $\rightarrow$ LUMO (78\%) \\
\hline LUMO & 4 & 2 & 50 & 45 & & \\
\hline номо & 32 & 2 & 33 & 33 & $3(326 \mathrm{~nm} f=0.0653)$ & HOMO-1 $\rightarrow$ LUMO (11\%) \\
\hline HOMO -1 & 5 & 1 & 47 & 47 & & HOMO $\rightarrow$ LUMO+2 (68\%) \\
\hline HOMO -2 & 15 & 1 & 42 & 42 & $4(320 \mathrm{~nm} f=0.1377)$ & HOMO -2 $\rightarrow$ LUMO (31\%) \\
\hline HOMO -3 & 29 & 4 & 33 & 34 & & HOMO -1 $\rightarrow$ LUMO+1(48\%) \\
\hline HOMO -4 & 22 & 1 & 39 & 38 & $5(319 \mathrm{~nm} \mathrm{f}=0.1153)$ & $\begin{array}{l}\text { HOMO }-2 \rightarrow \text { LUMO+1 (21\%) } \\
\text { HOMO-1 } \rightarrow \text { LUMO (36\%) } \\
\text { HOMO } \rightarrow \text { LUMO+2 (17\%) }\end{array}$ \\
\hline
\end{tabular}

Table S6. A description of the calculated MO contributions, excited state descriptions and their associated transitions for $\left[\operatorname{lr}\left(\mathbf{L}^{\mathbf{7}}\right)_{2}(\text { bipy })\right]^{+}$complex $(\mathrm{Q} 1$ and $\mathrm{Q} 2$ are the different quinoxaline ligands; bipy = bipyridine).

\begin{tabular}{|c|c|c|c|c|c|c|}
\hline \multirow[b]{2}{*}{ Orbital } & \multicolumn{4}{|c|}{ Moiety Contribution to Orbital (\%) } & \multicolumn{2}{|c|}{ Orbital Contribution to Excited State } \\
\hline & $\operatorname{lr}(5 d)$ & bipy & Q1 & Q2 & Excited State & Contributing Transitions (> 10\%) \\
\hline LUMO +4 & 1 & 29 & 35 & 35 & $1(390 \mathrm{~nm} f=0.2120)$ & HOMO-1 $\rightarrow$ LUMO (12\%) \\
\hline LUMO +3 & 2 & 51 & 23 & 24 & & HOMO $\rightarrow$ LUMO+1 (80\%) \\
\hline LUMO +2 & 2 & 96 & 1 & 1 & $2(385 \mathrm{~nm} f=0.0677)$ & $\begin{array}{l}\text { HOMO-1 } \rightarrow \text { LUMO+1 (15\%) } \\
\text { HOMO } \rightarrow \text { LUMO (80\%) }\end{array}$ \\
\hline LUMO +1 & 4 & 1 & 54 & 41 & & \\
\hline LUMO & 4 & 1 & 41 & 54 & & \\
\hline HOMO & 33 & 2 & 32 & 32 & $3(324 \mathrm{~nm} f=0.2233)$ & $\begin{array}{l}\text { HOMO-2 } \rightarrow \text { LUMO+1 (26\%) } \\
\text { HOMO-1 } \rightarrow \text { LUMO (46\%) }\end{array}$ \\
\hline HOMO -1 & 6 & 1 & 47 & 47 & & HOMO $\rightarrow$ LUMO+2 (12\%) \\
\hline HOMO -2 & 12 & 1 & 44 & 43 & $4(323 \mathrm{~nm} \mathrm{f}=0.1541)$ & $\begin{array}{l}\text { HOMO }-2 \rightarrow \text { LUMO (29\%) } \\
\text { HOMO }-1 \rightarrow \text { LUMO+1(52\%) }\end{array}$ \\
\hline HOMO -3 & 46 & 8 & 23 & 23 & & \\
\hline HOMO -4 & 49 & 3 & 24 & 24 & $5(317 \mathrm{~nm} \mathrm{f}=0.0201)$ & HOMO $\rightarrow$ LUMO+2 (75\%) \\
\hline
\end{tabular}


Table S7. A description of the calculated MO contributions, excited state descriptions and their associated transitions for $\left[\operatorname{Ir}\left(\mathrm{L}^{\mathbf{8}}\right)_{2}(\text { bipy })\right]^{+}$complex $(\mathrm{Q} 1$ and $\mathrm{Q} 2$ are the different quinoxaline ligands; bipy = bipyridine).

\begin{tabular}{|c|c|c|c|c|c|c|}
\hline \multirow[b]{2}{*}{ Orbital } & \multicolumn{4}{|c|}{$\begin{array}{c}\text { Moiety Contribution to } \\
\text { Orbital (\%) } \\
\end{array}$} & \multicolumn{2}{|c|}{ Orbital Contribution to Excited State } \\
\hline & $\operatorname{Ir}(5 d)$ & bipy & Q1 & Q2 & Excited State & Contributing Transitions (> 10\%) \\
\hline LUMO +4 & 1 & 15 & 42 & 42 & $1(377 \mathrm{~nm} \mathrm{f}=0.1679)$ & HOMO-1 $\rightarrow$ LUMO (11\%) \\
\hline LUMO +3 & 2 & 65 & 17 & 17 & & HOMO $\rightarrow$ LUMO+1 (81\%) \\
\hline LUMO +2 & 2 & 97 & 1 & 1 & $2(377 \mathrm{~nm} \mathrm{f}=0.0416)$ & HOMO $\rightarrow$ LUMO (84\%) \\
\hline LUMO +1 & 4 & 1 & 47 & 48 & & \\
\hline LUMO & 3 & 2 & 48 & 47 & & \\
\hline HOMO & 34 & 2 & 32 & 32 & $3(318 \mathrm{~nm} \mathrm{f}=0.1685)$ & HOMO-2 $\rightarrow$ LUMO+1 (28\%) \\
\hline HOMO -1 & 4 & 1 & 48 & 48 & & $\begin{array}{l}\text { HOMO-1 } \rightarrow \text { LUMO (35\%) } \\
\text { HOMO } \rightarrow \text { LUMO+2 (21\%) }\end{array}$ \\
\hline HOMO -2 & 8 & 1 & 46 & 45 & $4(316 \mathrm{~nm} f=0.1725)$ & HOMO-2 $\rightarrow$ LUMO (43\%) \\
\hline HOMO -3 & 20 & 4 & 38 & 38 & & HOMO-1 $\rightarrow$ LUMO+1(41\%) \\
\hline HOMO -4 & 17 & 0 & 42 & 41 & $5(318 \mathrm{~nm} f=0.0666)$ & HOMO $\rightarrow$ LUMO+2 (64\%) \\
\hline
\end{tabular}

Table S8. A description of the calculated MO contributions, excited state descriptions and their associated transitions for $\left[\operatorname{lr}\left(\mathrm{L}^{\mathbf{9}}\right)_{2}(\text { bipy })\right]^{+}$complex $(\mathrm{Q} 1$ and $\mathrm{Q} 2$ are the different quinoxaline ligands; bipy = bipyridine).

\begin{tabular}{|c|c|c|c|c|c|c|}
\hline \multirow[b]{2}{*}{ Orbital } & \multicolumn{4}{|c|}{$\begin{array}{c}\text { Moiety Contribution to } \\
\text { Orbital (\%) }\end{array}$} & \multicolumn{2}{|c|}{ Orbital Contribution to Excited State } \\
\hline & $\begin{array}{l}\text { Ir } \\
\text { (5d) }\end{array}$ & bipy & Q1 & Q2 & Excited State & Contributing Transitions (> 10\%) \\
\hline LUMO +4 & 1 & 36 & 31 & 32 & $1(375 \mathrm{~nm} \mathrm{f}=0.2381)$ & HOMO-1 $\rightarrow$ LUMO (14\%) \\
\hline LUMO +3 & 2 & 77 & 11 & 11 & & HOMO $\rightarrow$ LUMO+1 (78\%) \\
\hline LUMO +2 & 2 & 97 & 1 & 1 & $2(374 \mathrm{~nm} \mathrm{f}=0.0665)$ & HOMO-1 $\rightarrow$ LUMO+1 (13\%) \\
\hline LUMO +1 & 4 & 2 & 46 & 47 & & HOMO $\rightarrow$ LUMO (82\%) \\
\hline LUMO & 3 & 2 & 48 & 47 & & \\
\hline номо & 32 & 2 & 33 & 33 & $3(322 \mathrm{~nm} \mathrm{f}=0.1380)$ & HOMO-2 $\rightarrow$ LUMO+1 (22\%) \\
\hline HOMO -1 & 2 & 1 & 49 & 49 & & $\begin{array}{l}\text { HOMO-1 } \rightarrow \text { LUMO (24\%) } \\
\text { HOMO } \rightarrow \text { LUMO+2 (39\%) }\end{array}$ \\
\hline HOMO -2 & 9 & 1 & 45 & 45 & $4(319 \mathrm{~nm} f=0.1791)$ & HOMO-2 $\rightarrow$ LUMO (47\%) \\
\hline HOMO -3 & 20 & 3 & 38 & 38 & & HOMO-1 $\rightarrow$ LUMO+1(38\%) \\
\hline HOMO -4 & 11 & 0 & 45 & 44 & $5(318 \mathrm{~nm} \mathrm{f}=0.1056)$ & $\begin{array}{l}\text { HOMO-4 } \rightarrow \text { LUMO+1 (20\%) } \\
\text { HOMO-3 } \rightarrow \text { LUMO (32\%) } \\
\text { HOMO } \rightarrow \text { LUMO+2 (27\%) }\end{array}$ \\
\hline
\end{tabular}


Table S9. A description of the calculated MO contributions, excited state descriptions and their associated transitions for $\left[\operatorname{lr}\left(\mathrm{L}^{10}\right)_{2}(\text { bipy })\right]^{+}$complex $(\mathrm{Q} 1$ and Q2 are the different quinoxaline ligands; bipy = bipyridine).

\begin{tabular}{l|cccc|ll}
\hline & \multicolumn{3}{|c|}{$\begin{array}{c}\text { Moiety Contribution to } \\
\text { Orbital (\%) }\end{array}$} & \multicolumn{3}{c}{ Orbital Contribution to Excited State } \\
\hline Orbital & Ir (5d) & bipy & Q1 & Q2 & Excited State & Contributing Transitions (> 10\%) \\
\hline \hline LUMO +4 & 1 & 11 & 45 & 44 & $1(381 \mathrm{~nm} \mathrm{f=0.1896)}$ & HOMO-1 $\rightarrow$ LUMO (11\%) \\
LUMO +3 & 2 & 54 & 22 & 23 & & HOMO $\rightarrow$ LUMO+1 (81\%) \\
LUMO +2 & 2 & 96 & 1 & 1 & $2(380 \mathrm{~nm} \mathrm{f=0.0476)}$ & HOMO-1 $\rightarrow$ LUMO+1 (11\%) \\
LUMO +1 & 4 & 1 & 48 & 48 & & HOMO $\rightarrow$ LUMO (84\%) \\
LUMO & 3 & 2 & 47 & 48 & & \\
HOMO & 33 & 2 & 33 & 33 & $3(321 \mathrm{~nm} \mathrm{f=0.2982)}$ & HOMO-2 $\rightarrow$ LUMO+1 (36\%) \\
HOMO -1 & 2 & 1 & 49 & 49 & & HOMO-1 $\rightarrow$ LUMO (42\%) \\
HOMO -2 & 6 & 1 & 47 & 46 & $4(320 \mathrm{~nm} \mathrm{f=0.2145)}$ & HOMO-2 $\rightarrow$ LUMO (44\%) \\
HOMO -3 & 16 & 3 & 41 & 41 & & HOMO-1 $\rightarrow$ LUMO+1(40\%) \\
HOMO -4 & 21 & 4 & 38 & 38 & $5(312 \mathrm{~nm} \mathrm{f=0.0163)}$ & HOMO $\rightarrow$ LUMO+2 (81\%) \\
\hline
\end{tabular}
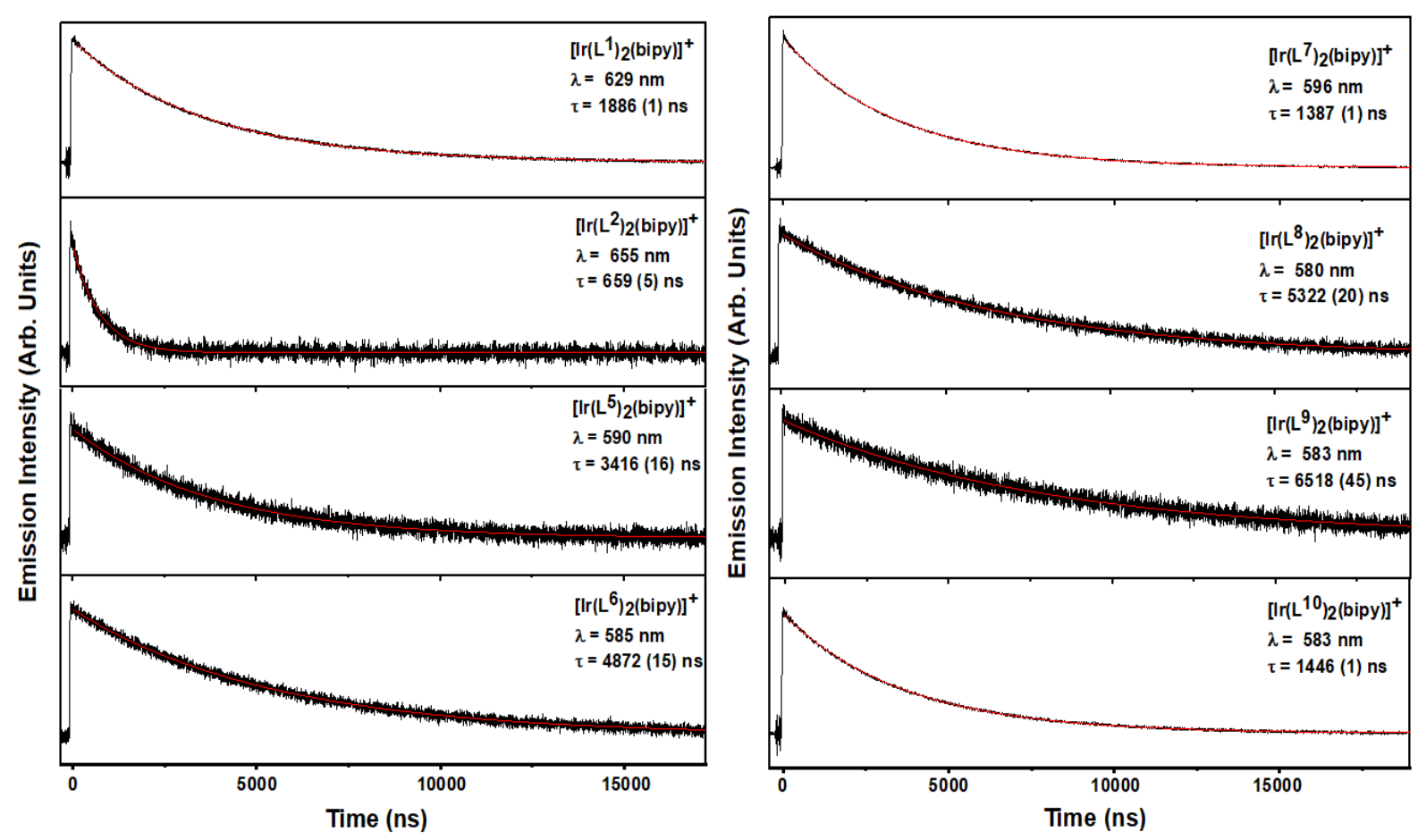

Figure S10. Fitted kinetic decays of the emission feature of each complex under deoxygenated conditions (MeCN). 


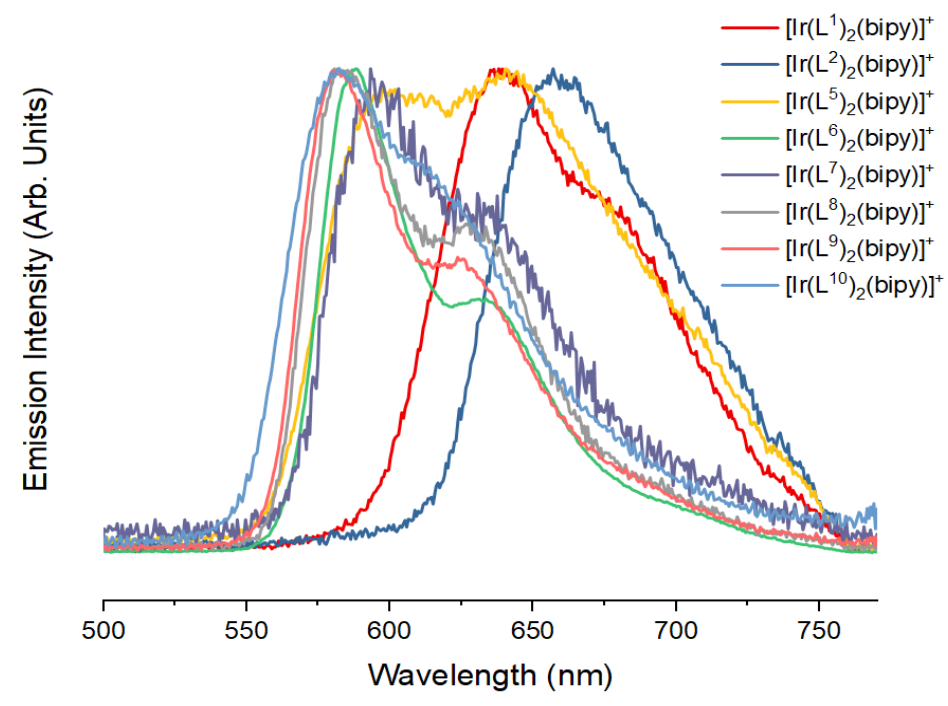

\begin{tabular}{|c|c|}
\hline Complex & $\begin{array}{r}77 \mathrm{~K} \\
\tau / \mathrm{ns}^{\mathrm{d}} \\
\end{array}$ \\
\hline$\left[\operatorname{Ir}\left(\mathbf{L}^{1}\right)_{2}(\right.$ bipy $\left.)\right] \mathrm{PF}_{6}$ & 2691 \\
\hline$\left[\operatorname{lr}\left(\mathrm{L}^{2}\right)_{2}(\right.$ bipy $\left.)\right] \mathrm{PF}_{6}$ & 2362 \\
\hline$\left[\operatorname{lr}\left(\mathbf{L}^{5}\right)_{2}(\right.$ bipy $\left.)\right] \mathrm{PF}_{6}$ & 1795 \\
\hline$\left[\operatorname{lr}\left(\mathrm{L}^{6}\right)_{2}(\right.$ bipy $\left.)\right] \mathrm{PF}_{6}$ & 3636 \\
\hline$\left[\operatorname{lr}\left(\mathrm{L}^{7}\right)_{2}(\right.$ bipy $\left.)\right] \mathrm{PF}_{6}$ & 4210 \\
\hline$\left[\operatorname{lr}\left(\mathrm{L}^{8}\right)_{2}(\right.$ bipy $\left.)\right] \mathrm{PF}_{6}$ & 4818 \\
\hline$\left[\operatorname{lr}\left(\mathrm{L}^{9}\right)_{2}(\right.$ bipy $\left.)\right] \mathrm{PF}_{6}$ & 5184 \\
\hline$\left[\operatorname{Ir}\left(\mathrm{L}^{10}\right)_{2}(\right.$ bipy $\left.)\right] \mathrm{PF}_{6}$ & 4306 \\
\hline
\end{tabular}

Figure S11. Normalized emission spectra recorded for all isolated $\operatorname{Ir}(\mathrm{III})$ complexes (77 K, MeCN glass). Corresponding lifetimes shown tabulated, right.

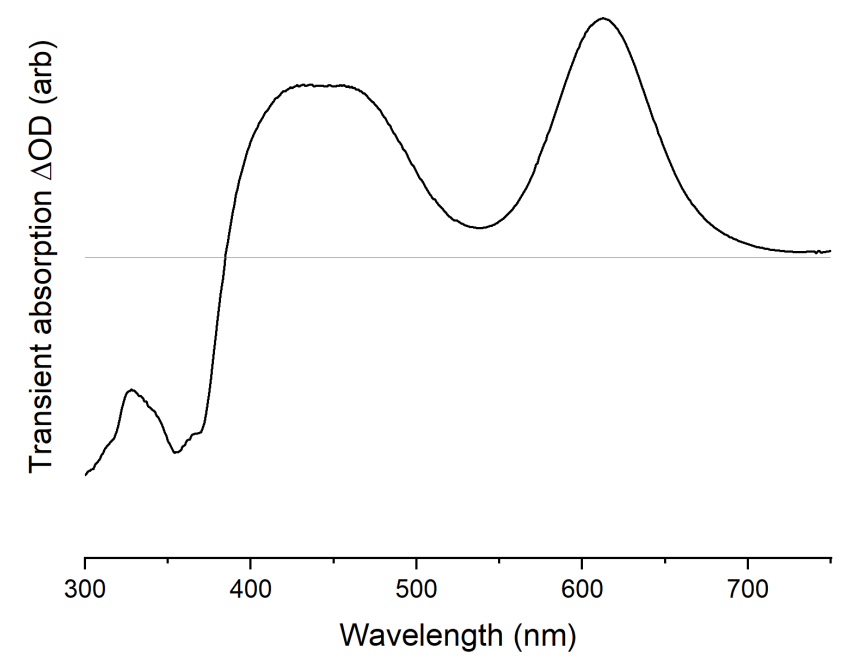

Figure S12. Simulated TA spectrum for $\left[\operatorname{lr}\left(L^{8}\right)_{2}(\text { bipy })\right]^{+}$. 

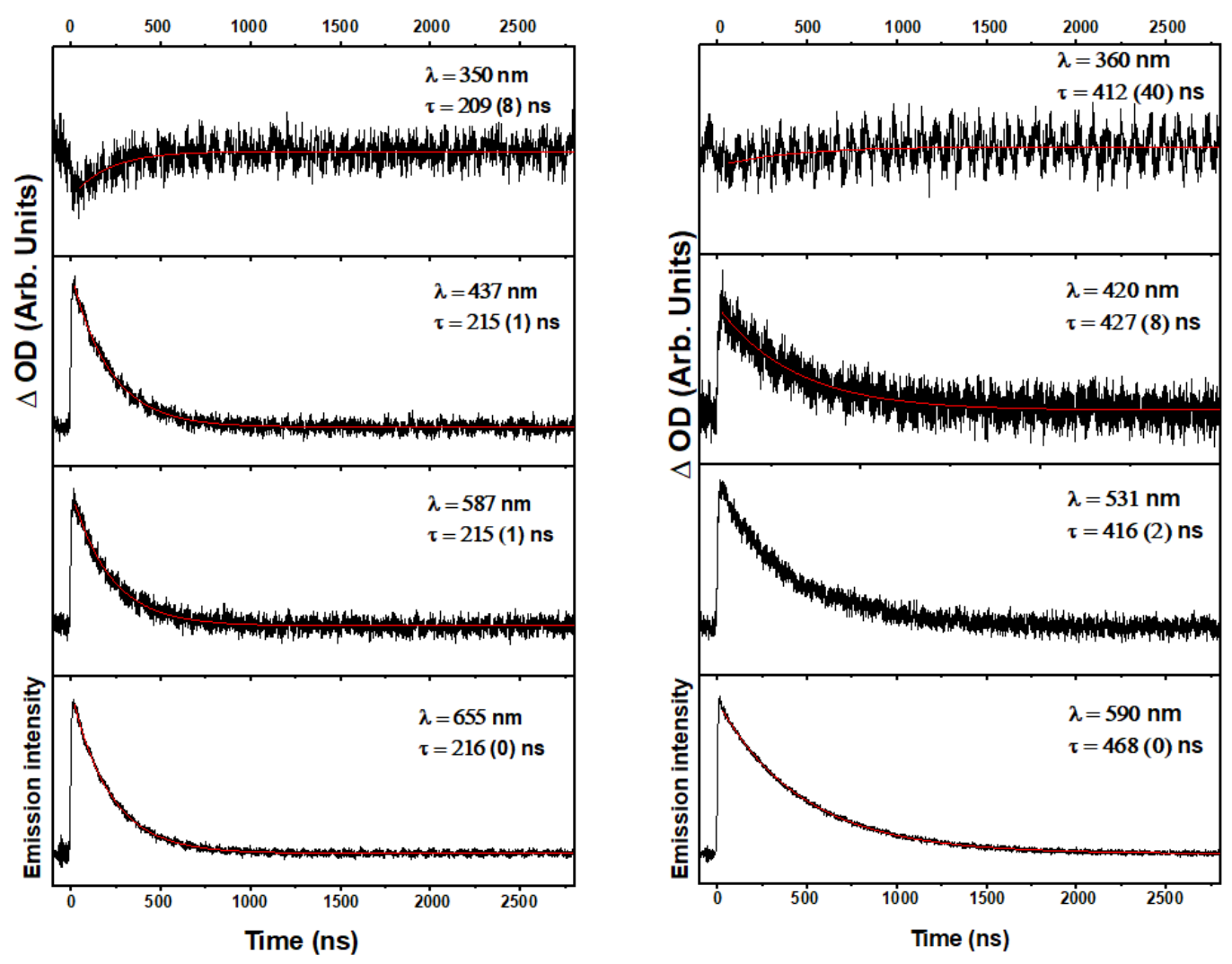

Figure S13. Kinetic traces of the major features of the transient spectra of $\left[\operatorname{Ir}\left(\mathbf{L}^{2}\right)_{2}\right.$ (bipy)]PF 6 (left) and $\left[\operatorname{Ir}\left(\mathbf{L}^{5}\right)_{2}(\right.$ bipy $\left.)\right] \mathrm{PF}_{6}$ (right) complexes in acetonitrile at room temperature, aerated. Wavelengths and lifetimes of each trace are inset. 

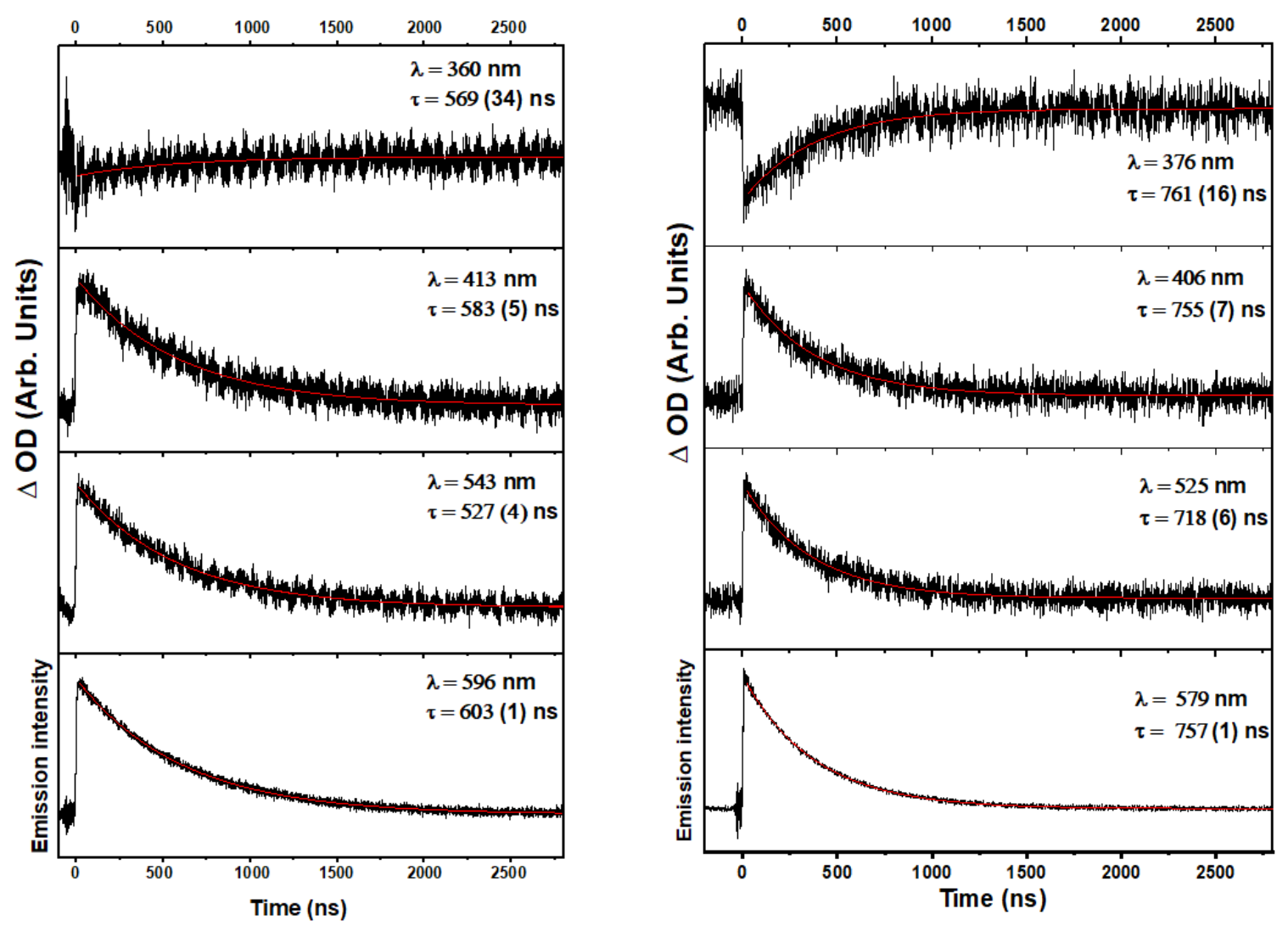

Figure S14. Kinetic traces of the major features of the transient spectra of

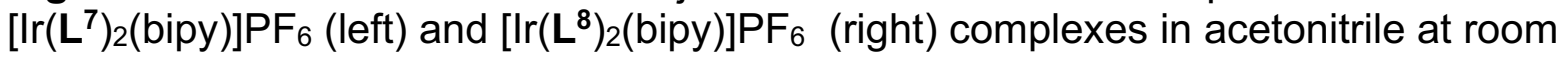
temperature, aerated. Wavelengths and lifetimes of each trace are inset. 

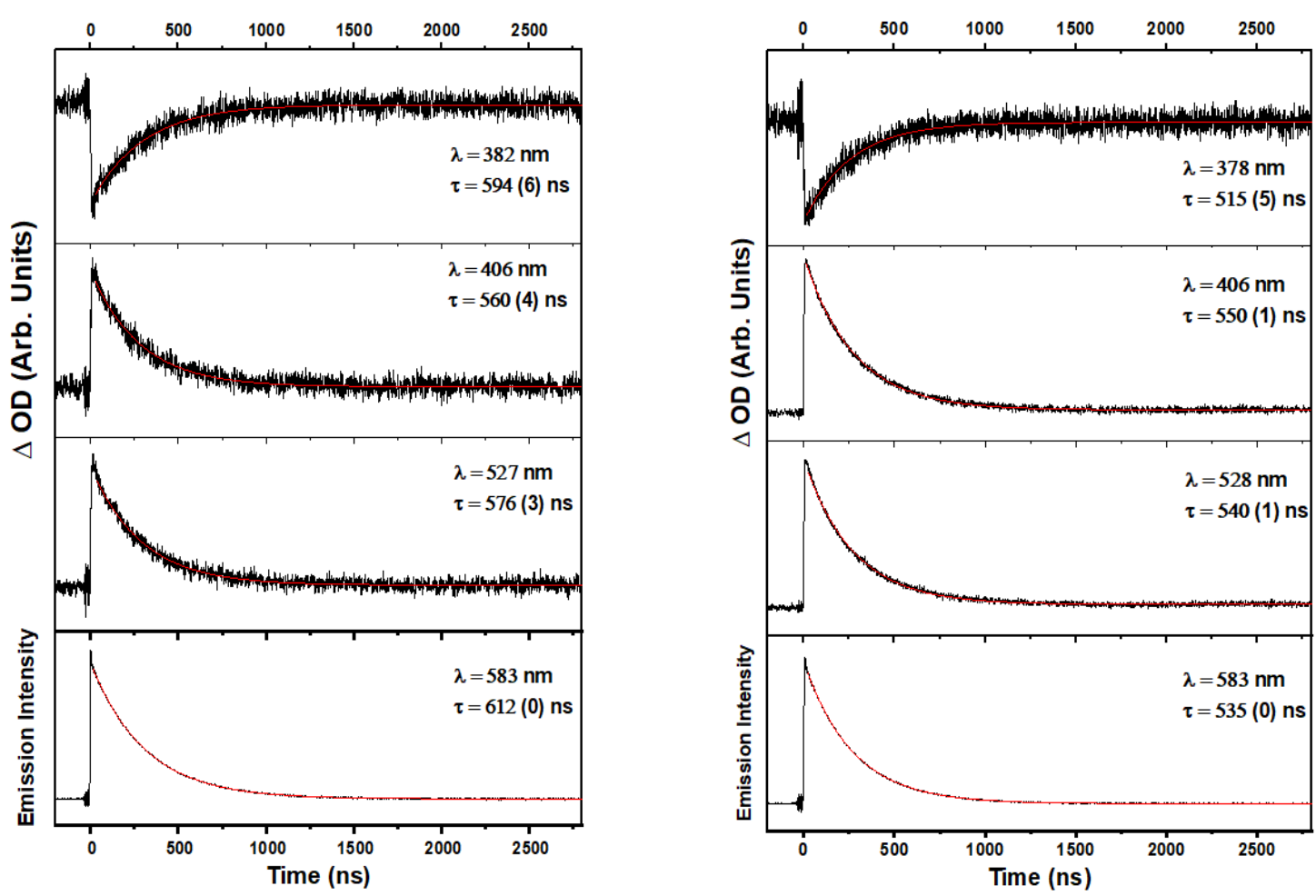

Figure S15. Kinetic traces of the major features of the transient spectra of $\left[\operatorname{Ir}\left(\mathbf{L}^{9}\right)_{2}\right.$ (bipy)]PF 6 (left) and $\left[\operatorname{Ir}\left(\mathbf{L}^{10}\right)_{2}\right.$ (bipy)]PF 6 (right) complexes in acetonitrile at room temperature, aerated. Wavelengths and lifetimes of each trace are inset. 